This item was submitted to Loughborough's Research Repository by the author.

Items in Figshare are protected by copyright, with all rights reserved, unless otherwise indicated.

\title{
Flow properties of an intact MPL from nano-tomography and pore network modelling
}

\section{PLEASE CITE THE PUBLISHED VERSION}

http://dx.doi.org/10.1016/j.fuel.2014.07.040

\section{PUBLISHER}

(C) Elsevier

\section{VERSION}

AM (Accepted Manuscript)

\section{PUBLISHER STATEMENT}

This work is made available according to the conditions of the Creative Commons Attribution-NonCommercialNoDerivatives 4.0 International (CC BY-NC-ND 4.0) licence. Full details of this licence are available at: https://creativecommons.org/licenses/by-nc-nd/4.0/

\section{LICENCE}

CC BY-NC-ND 4.0

\section{REPOSITORY RECORD}

Ma, Jingsheng, Xiaoxian Zhang, Zeyung Jiang, Hossein Ostadi, Kyle Jiang, and Rui Chen. 2015. "Flow Properties of an Intact MPL from Nano-tomography and Pore Network Modelling". figshare. https://hdl.handle.net/2134/16560. 


\section{Flow properties of an intact MPL from nano-tomography and pore network modelling}

Jingsheng Ma ${ }^{\mathrm{a}}$, Xiaoxian Zhang ${ }^{\mathrm{b}}$, Zeyung Jiang ${ }^{\mathrm{a}}$, Hossein Ostadi ${ }^{\mathrm{c}}$, Kyle Jiang ${ }^{\mathrm{c}}$ and Rui Chen ${ }^{\mathrm{d}}$

8 a Institute of Petroleum Engineering, Heriot-Watt University, Edinburgh, EH14 4AS UK.

$9 \quad$ UK. e-mail: Jingsheng.Ma@ pet.hw.ac.uk; Zeyung.Jiang@ pet.hw.ac.uk

$10{ }^{\mathrm{b}}$ School of Engineering, University of Liverpool, Brownlow Street, Liverpool, L69 3GQ UK.

11 e-mail: Xiaoxian.zhang@ liverpool.ac.uk

$12{ }^{\mathrm{c}}$ Department of Mechanical and Manufacturing Engineering, University of Birmingham,

13 BirminghamB15 2TT, UK. e-mail: ostadi@ contacs.bham.ac.uk; k.jiang@ bham.ac.uk

$14{ }^{\mathrm{d}}$ Department of Aeronautical and Automotive Engineering, Loughborough University,

15 Leicestershire LE11 3TU, UK. e-mail: $\underline{\text { R.Chen@1boro.ac.uk }}$

18 Corresponding author;

19 Dr. Jingsheng Ma, Institute of Petroleum Engineering, Heriot-Watt University, Edinburgh, EH14 20 4AS UK.

$21 \quad$ Tel: +441314518296

22 E-mail: Jingsheng.Ma@pet.hw.ac.uk; 


\section{Abstract}

Adding a hydrophobic micro-porous layer (MPL) between a gas diffusion layer (GDL) and a catalyst layer (CL) at the cathode of a PEM fuel cell was found capable of improving cell performance. However, how an MPL does this is not well-understood because current techniques are limited in measuring, observing and simulating multiphase pore fluid flow across the full range of pores that vary to a great extent in geometry, topology, surface morphology. In this work, we focused our investigation on estimating flow properties of an MPL volume to assess the limiting effect of strongly hydrophobic sub-micron pores on water transports. We adopted a nanotomography and pore network flow modelling approach. A pore-structure model, purposely reconstructed from an intact MPL sample using Focused Ion Beam milling and Scanning Electron Microscope (FIB/SEM) previously, was used to extract a realistic pore network. A two-phase pore network flow model, developed recently for simulating the flow of gas, liquid or their mixture in both micrometre and nanometre pores, was applied to the pore network. We firstly tested the validity of the constructed pore network, and then calculated the properties: permeability for both water and selected gases, water entry pressure, and relative permeability. Knudsen diffusion was taken into consideration in calculations when appropriate. Our calculations showed that the water permeability was three orders of magnitude smaller than experimentally measured results reported in the literature, and when the water contact angle increased from $95^{\circ}$ to $150^{\circ}$, the water-entry pressure increased from 2.5 $\mathrm{MPa}$ to $28 \mathrm{MPa}$. Thus our results revealed that for a strongly hydrophobic MPL that contains nanometre pores only it would behave like a buffer to water, and therefore the structural preferential paths in an MPL, such as cracks, are likely to be responsible for significant liquid water transport from the CL to the GDL that has been observed experimentally recently. We highlighted the needs for multi-scale modelling of the interplays of liquid water and gas transfer in MPLs that contain variable pores.

Key words: PEM fuel cells; micro-porous layer; FIB/SEM tomography; pore network flow modelling; liquid water flow. 


\section{Introduction}

The polymer exchange membrane (PEM) fuel cell has been touted as a promising alternative to combustion engine due to its high efficiency, low emission and sustainable availability of hydrogen. Since the PEM fuel cell operates at a low temperature, the water vapour it produces at the cathode could condense when working at high current density. Managing the condensed water is hence a critical issue. On the one hand, the electrolyte membrane needs to be hydrated in order to facilitate proton movement, and this is commonly done by humidifying the hydrogen at the anode. On the other hand, the condensed water at the cathode needs to be removed from the cell to avoid flooding. Humidifying hydrogen at the anode and removing water from the cathode are self-defeating, and an improved design is hence required to effectively manage the water [1].

Recent research found that adding a micro-porous layer (MPL) between a gas diffusion layer (GDL) and a catalyst layer (CL) at the cathode can enhance fuel cell performance $[2,3]$ because of the improvement in water management. The MPL is highly hydrophobic and functions as a capillary barrier, forcing the condensed water in the CL to build up a sufficiently high pressure before it can move into it [4]. On the other hand, the CL is less hydrophobic than the MPL and the increased pressure in it can drive the water moving backwards into the membrane so as to keep it hydrated [5]. Apart from improving water management, the MPL also increases the CL and GDL contact and reduces the damage caused to the CL by the clamping force applied to the fuel cell assembly. However, the MPL has some detrimental impacts: 1) it makes oxygen difficult to move into the CL because it is less permeable than the GDL; and 2) the increased water pressure in the CL could block the pathways of gaseous oxygen. These conflicting effects reveal that the MPL needs to be optimally designed [6], and this, in turn, calls for a better understanding how different pores in an MPL influence fluid transports.

Markedly different pore systems may be formed in MPLs depending on methods used to fabricate them and to assemble them into fuel cells. Fabrication methods may differ in the selection 
of forming agents and their composition, MPL forming process (e.g. composites mixing, drying and sintering) and techniques for applying an MPL to a GDL (see [7-9] and references therein). Both imaging and laboratory measuring techniques have been used to characterise the pore systems of MPLs and results reported in the literature suggest that an MPL contains three classes of pores: pores at nanometre scales, pores at micrometre scales and cracks, while their proportion can vary from one MPL to another greatly [7, 10, 11]. SEM images of MPLs, which are commonly fabricated using carbon black and polytetrafluoroethylene (PTFE), show that they typically contain a larger number of nanometre pores than micrometre pores, and cracks of variable apertures up to tens of microns [11-13]. The fabrication can induce cracks whose geometrical characters and spatial patterns may be influenced by many factors including the hydrophobicity and thickness of an MPL and drying and sintering conditions [14]. For other MPLs fabricated from carbon black and poly(vinylidene fluoride) (PVDE) for example, they may contain much more micrometre pores than their PTFE counterparts do but none or very few of cracks [7]. Besides those formed during fabrication, cracks are known to be induced during the assembly of fuel cell components under mechanical loads and operations of wet-dry cycles $[15,16]$.

Over the past few years, advances in imaging technology such as X-ray computed tomography (XCT) and FIB/SEM enable one to visualise the interior structures of a GDL, an MPL, a CL or an assembly of them at a resolution ranging from a few tens of microns down to a few tens of nanometres [17-19]. Therefore these techniques allow one to characterise MPL pore geometry, topology and surface morphology quantitatively and to develop pore-space models on which to simulate multi-phase flow and to estimate flow properties. Because MPL pores are diverse in size and shape, and every imaging technique has a limited field view, it is not possible to obtain 'a representative element pore volume' of the sample by any technique alone. For example, FIB/SEM and nano-XCT are ideal for imaging MPL nanometre pores on a small sample of a few tens of microns in dimension [20]. On the other hand, a micro-XCT is ideal for imaging a larger volume of 
104 a fuel cell assembly up to a few centimetres in dimension at a resolution adequate for revealing 105 micrometre pores and cracks $[17,18]$.

106 In conjunction with imaging-based pore characterisation, X-ray and neutron radiography 107 imaging techniques have been used to observe multiphase fluid displacements in a single or 108 multiple fuel cell units to examine qualitatively gas and liquid water transports $[10,21,22]$. Due to 109 their limiting spatial resolutions, these techniques have been exploited to the most effective for 110 visualising water movement in large pores or along cracks and the build-up of water saturation 111 along an MPL. Using soft X-ray radiography, Deevanhxay et al. [10, 23] observed water movement 112 preferential along cracks of an MPL and concluded that the MPL plays a crucial role in water 113 management.

114 Many laboratory tests have been designed to characterise pore structures and transport 115 properties of individual fuel cell components or an assembly of them. By measuring injection 116 pressure and injected fluid volume, a gas or mercury intrusion test on a unit can measure the pore 117 volume, surface area, size distribution and connectivity as well as capillary pressure of it [11]. 118 Multi-phase flow experiments may be extended to measure liquid and gas permeability and the 119 relative permeability of gas and liquid for MPLs [24, 25]. These tests have their limitations too. 120 Fluid intrusion tests are often insufficient to discriminate normal pores from cracks. In flow 121 experiments, the permeability and relative permeability characterise the overall flow of a measured 122 sample but cannot be attributed to different types of pores. Consequently, the experimental results 123 have limited use for designing optimal MPLs. It should be noted that all laboratory tests are difficult 124 to apply to an individual MPL because it is too small. As a result, the effect of an MPL has to be 125 inferred from multiple tests carried out on samples with and without it.

126 Numerical modelling techniques have been developed and applied to study fluid flow 127 behaviours in MPLs and GDLs. Pasaogullari and Wang [26] developed a multi-phase mixture 128 model. Unlike other continuum models based on the unsaturated flow theory, their model considers 
129 the gas-phase flow countering capillarity-induced liquid water motion, and, therefore, enables those 130 authors to elucidate the effects of porosity, thickness and wettability of an MPL on the two-phase 131 flow more appropriately. They found that the optimal thickness of an MPL is 30 to 45 microns for 132 water management. However, the validity of the continuum models is questionable. Rebai and Prat 133 [27] argued that even a GDL is too small to be considered as a representative elementary volume 134 (REV) and showed that the two-phase flow properties become size-dependent rather than size135 independent that would be expected for a REV and also assumed in the continuum models. They 136 concluded that pore-scale modelling, in particular pore-network flow modelling, is the most 137 appropriate approach.

138 In conjunction with image-based pore characterisation and the pore-scale reconstruction of 139 MPL volumes, some recent work adopted pore-scale flow models to estimate fluid flow properties. 140 Ostadi et al. [17] reconstructed a model from a small intact MPL volume using FIB/SEM, and they 141 then estimated its phase volume fractions, pore connectivity, tortuosity and pore size distribution, 142 and calculated its fluid flow properties including the diffusivity coefficient and permeability. Wargo 143 et al. [18] took a similar approach and reconstructed models for an intact MPL volume to, in 144 addition, characterise capillary pressure-saturation relationship; it shows that a strongly 145 hydrophobic MPL forms a good capillary barrier to water. Both groups estimated permeability 146 values for their respective MPL models using a lattice Boltzmann method and their results are in a 147 good agreement with each other. As a lattice Boltzmann method is applied to a voxel-based binary 148 image model directly, it can account for the full geometric complicity of that model. However, two149 phase simulation on a binary image is not only much more time consuming than a single-phase 150 simulation but also prone to numerical instability for high-density contrasted phases like gas and 151 liquid water. Although latest modifications have improved the stability of the two-phase lattice 152 Boltzmann models, they require time-consuming calculations of second-order derivatives [28, 29]. 
153 In addition, the standard lattice Boltzmann models could not account for the Knudsen diffusion 154 appropriately in small pores [30].

155 Pore network flow modelling is another approach to study the multiphase fluid flow at the pore 156 scales. There the pore space is simplified into connected network of node and bond elements on 157 which multiphase flow can be simulated efficiently. High contrasts in fluid density and viscosity do 158 not cause numerical stability while the slip flow and Knudsen diffusion can be modelled too. This 159 approach has been applied to study gas and water flow in PEM fuel cell, where void space is 160 simplified as spherical or cubic pores connected by cylinders with circular or rectangular cross161 sections on a regular lattice $[31,32]$ and to model the interactions of complex coupled processes 162 [33]. On the other hand, this approach underwent a substantial development over past two decades 163 in the fields of soil sciences and petroleum engineering. In recent years, the techniques have been 164 developed for extracting a pore network from a 3D binary image [34] and simulating multiphase 165 displacements on that network efficiently [35]. One of the emphases of the recent improvements has 166 been to capture the effect of complex geometry and topology of pore structures on multi-phase fluid 167 flow processes. This approach has been shown to be predictive in estimating multi-phase flow 168 properties when applied to natural soil and carbonate rocks, and can therefore be used to gain 169 accurate predictions of fluid flow properties for MPLs.

170 It is, however, difficult to quantify fluid flow of an MPL sample using any type of the 171 techniques alone if its pore structure is composed of multi-scale pores varying greatly in space. For 172 example, cracks have been postulated to offer preferential pathways for water, but laboratory tests 173 have not been able to confirm this unambiguously and to explain the precise mechanisms that an 174 MPL improves the cell performance. Imaging-based experimental observations may reveal the 175 existence of crack networks in an MPL and liquid water flowing through its cracks into a GDL, but 176 are insufficient to explain the emergent water saturations away from cracks [10]. Therefore, a better 177 understanding of the role of MPLs in water management may be gained by pore network modelling 
to quantify the contributions of different pore types (e.g. nanometre, micrometre or crack pores) to gas and water transports, respectively and collaboratively.

This paper reports a pore network modelling study of an intact MPL to estimate the likely contribution of nanometre pores alone to gas and water transports. It is organised as follows. Section 2 describes the methodology this work took, namely FIB/SEM imaging, pore-network construction and a pore-network flow modelling. Section 3 presents the results and analyses, followed by a section of discussion and remarks. It argues that MPL cracks contribute mainly to the water flow if an MPL contains cracks and pre-dominantly nano-scale pores and that it is important to take a multi-scale approach when coming to model fluid flow interactions within an MPL that contains cracks, micrometre and nanometre pores. Section 5 concludes this work.

\section{Methodology}

\subsection{FIB/SEM imaging}

The details of the FIB/SEM tomography used to acquire the microstructure of an MPL were given in our previous work [17]. We briefly describe it below for completeness.

A thin slice of approximately 14 nanometres wide on the side-wall of a trench inside an MPL (provided by Johnson Matthey Fuel Cells Ltd., UK) was milled away using FIM $\left(\mathrm{Ca}^{+}\right)$first. The exposed structure was then captured using SEM. Repeating this procedure produced a number of 2D slices. To protect the soft surface of the MPL from ion bombardment and pollution caused by FIB-induced re-decomposition of the precursor gases, the surface was coated by $100 \mathrm{~nm}$-thick layer of platinum prior to the side-wall milling. Each 2D image was processed by choosing a threshold to digitize it into a binary image with 0 for pore and 1 for solid. A procedure was employed to ensure the segmentation of pores and solids as accurate as possible. Stacking all these binary 2D images yields a 3D structure as shown in Figure 1.

For this sample, all pores are well-resolved by FIB/SEM at the acquisition resolution. In fact the pores are over-resolved as pore diameters are greater than $9 \mathrm{~nm}$ and $18 \mathrm{~nm}$ for more than $99.99 \%$ 
and $99.8 \%$ of all pores. For the purpose of reconstructing an image-based model with as many as cubic voxels in each direction, which is needed for accurately estimating pore geometry for a pore network, we re-sampled the segmented image at the voxel resolution of $8 \mathrm{~nm}$ in all 3 directions. From that re-sampled image, a volume of $149^{3}$ voxels was obtained and used in this work.

To ensure the selected volume large enough to be representative, we examined the variability of the porosity across sub-volumes. We randomly drew over 100 unique cubic sub-volumes at dimensions, ranging from 30 to 140 voxels with multiple volumes for each dimension. For each volume we calculated its porosity. The mean of the porosity values at each selected dimension is plotted against the dimension with one standard deviation being drawn as errors around the mean as shown in Figure 3. The figure shows that when the dimension increases above 100 voxels the mean porosity converges to that of the full volume (just below 34\%) with a fast decreasing error. It can

214 also be shown that when the dimension is greater than 120 , the relative difference of the mean 215 porosity is smaller than $2 \%$. Based on these results, we consider the selected volume to be large 216 enough and representative.

\subsection{Pore network extraction and pore network flow modelling}

A set of techniques developed by Jiang et al [34] was applied to extract a pore network from the selected volume. Here a Euclidean distance map for every pore voxel is calculated first, followed by a medial axial transformation to identify the skeletons of the pore space; then both the distance map and the skeletons are used to partition pore space into node space, while the remaining pore space, between each pair of nodes, into bond space; geometrical properties are calculated for every node and bond, including volume, hydraulic radius and shape factor. A shape factor is defined as the ratio of the area and the squared perimeter of the cross-section for an element; it indicates that an element has a cross-section that resembles a triangle, rectangle or circle, if it falls between $(0$, 0.048], $(0.048,0.062]$ or $(0.062,0.08]$, respectively. For a more detailed discussion of the construction of a pore network from a 3D binary image, the reader is referred to Appendix A for the 
228 a brief description of the extraction procedure and the original publication [34] for a full description.

229 In this work the pore network was extracted using an in-house software package that implements

230 those techniques.

A pore network constructed as such makes it possible to simulate multiphase fluid flow

efficiently and to capture critical phase displacement behaviours in geometrically and topologically

233 complex pores. It has been shown that a pore network model can predict the relative permeability of

234 oil and water for reservoir rocks $[35,36]$. In this work, single and two phase fluid flows were

235 simulated on the extracted pore network using a pore network flow model, extended from the two-

236 phase pore network flow model of Valvatne and Blunt [36]. The key extensions were made

237 primarily in calculating the gas-phase conductance in order to deal with slip flow and Knudsen

238 diffusion as they affect both single- and two-phase flows and, therefore, apparent gas permeability

239 and relative permeability. Those extensions have been discussed in details in a separate publication

240 [37] and summarised briefly here. The gas conductance for each network element is defined by

241 Equation (1) to account for the effects of the slip flow and the Knudsen diffusion in the second and

242 third terms inside the bracket, respectively. The equation was developed for the element with a

243 circular cross-section first and then adapted for an element with a non-circular cross-section through

244 a correction factor $\mathrm{C}_{\mathrm{g}}$. The non-ideal gas effect was expressed using van der Waals's two-parameter

245 principle of corresponding states in coefficient terms NIG\#. Because the gas conductance is

246 pressure-dependent and the gas pressure equations are no longer linear, an iterative solution

247 procedure was developed. For a brief explanation of nomenclature in the equation, see Appendix B.

248 The reader is referred to the original publication for details.

$$
C_{N I G}=\frac{C_{g} R_{h}^{2} \rho_{2}}{\mu_{2}}\left\{\frac{\mu_{2}}{\mu_{0}} N I G_{c}+\frac{\mu_{2}}{\mu_{2}^{i g}} \frac{8}{3}\left(\frac{2-\alpha}{\alpha}\right) k_{n, 2 h}+\frac{\mu_{2}}{\mu_{2}^{i g}} \frac{64}{9 C_{g} \pi} N I G_{k} k_{n, 2 h}\right\} A
$$

The same phase displacement rules as given in [36] remain. Therefore, the extended model can 251 simulate capillary-dominated flow with insignificant viscous pressure drops across the model. The 
order of capillary domination is defined by the capillary number, the ratio between viscous to capillary forces, and should be around $10^{-4}$ or less. Under this condition, the phase interfaces through a connected pore network can be considered to be static and the phase displacement is defined a sequence of ordered events determined by the capillary pressure. The capillary pressure of an element, $p_{c}$, was calculated from the Young-Laplace equation, $p_{c}=p_{w}-p_{a}=\sigma\left(1 / r_{1}+1 / r_{2}\right)$, where $p_{\mathrm{a}}$ and $p_{\mathrm{w}}$ are the air and water pressures, respectively, $\sigma$ is the water-air interfacial tension, and $r_{1}$ and $r_{2}$ are the principal radii of the curvature of water-air interface in the element if it is invaded by the water. For an element with a circular cross section, its capillary pressure is $p_{c}=2 \sigma \cos (\theta) / r$, where $r$ is the radius of the cross section of the element while $\theta$ is the contact angle of the wetting phase measured from the solid to the fluid interface. For each element with a triangular, rectangular or circular cross-section, its capillary pressure can be determined analytically (see [36] and the references therein).

Note that equation (1) was applied to gas and liquid two-phase flow where the wetting gas occupies the corners of a pore element, or as gas film, and connects throughout pore volumes from 266 the inlet to the outlet. Note that gas can be present in corners only when the contact angle is 267 sufficiently high, and this condition was assumed to hold in this work because only strongly 268 hydrophobic pores are of concern. When the liquid occupies the centre of an element, each corner 269 was considered as an independent element with a variable volume that can be empirically 270 determined [38]. Because each of the corner volumes is smaller than the host whole volume of the 271 host element, the total Knudsen diffusion effect for that element is expected to be stronger in two272 phase than single-phase cases. In summary, the extended pore network flow model can simulate 273 single-phase flow of the water or a non-ideal gas, and the two-phase flow for the non-wetting water 274 phase displacing the wetting gas phase in gas filled pores. The gas slip flow at pore surface and 275 Knudsen diffusion in pores are taken into consideration when appropriate. 
In this work, we simulated single-phase flow for water and three individual gases and two-

277 phase displacement of the air by the water on the extracted pore network. In the single-phase simulations, we first simulated water flow under a pressure gradient in which only the continuum flow was considered and then calculated the water permeability. To show the impact of the Knudsen diffusion on the permeability for different gases, we simulated the gas flow, under applied 281 pressure gradients from $50 \mathrm{kPa} / \mathrm{m}$ to $350 \mathrm{kPa} / \mathrm{m}$ with the outlet pressure being fixed at $100 \mathrm{kPa}$, and 282 then calculated the apparent gas permeability for oxygen, helium and hydrogen, respectively. The gas pressures were chosen to be similar to the experimental settings used by Pant et al [24]. In the two-phase simulation, the MPL was initially dry and filled with the air. Then the liquid water was driven into the MPL by a pressure imposed at the inlet. The water percolated through a gas filled element from its centre quasi-statically. When the pressure is increased incrementally, the saturation and capillary pressure relationship can be determined. Because of the contrasting density of the air and the water, the impact of air-water friction on water flow was neglected in the simulations.

In both single- and two-phase simulations, we considered the Knudsen diffusion effect for any pore whose Knudsen number is greater than 0.1, and set parameter $\alpha$ in Equation (1) to be 1.0 to control gas slippage at pore walls (see comments on choosing values for this parameter in [37]).

\section{Result analysis}

Figure 2 shows the partitioning of the pore space. Figure 4 shows the distribution of the calculated shape factors for all elements in the extracted pore network. The pore network have 1317 pores and 3222 throats, in which 2028 are triangular throats, 1064 rectangular throats and 71 cylindrical throats. Note that to simplify the calculation of the capillary pressure for elements with irregular cross-sections, we follow the same approximation convention as in [36]; that is, in all simulations we treated every element as to have equilateral triangular, square or circular crosssection if its shape factor falls between $(0,0.048],(0.048,0.062]$ or $(0.062,0.08]$, respectively. To 
301 validate representativeness of this network and the simplification above, we calculated the water permeability in all three directions on the extracted network, and then compared them with the permeability calculated on the corresponding volume of the binary image using the lattice Boltzmann model we developed and validated previously $[39,40]$. The comparison is shown in Table 1. The results agree well, indicating that the extracted pore network is accurate enough to hydraulically represent the pore geometry in the MPL.

The MPL is highly hydrophobic with a water contact angle higher than $100^{\circ}$ [41]. As a result, the water can only enter the MPL from the CL after building up a water pressure so that the pressure difference between the water and air pressures $\Delta \mathrm{P}$, i.e. capillary pressure, excesses the water-entry pressure at each pore. The value of the water-entry pressure depends on the geometry of each pore and its hydrophobicity, which is represented by the water contact angle [41]. The change of the water-entry pressure with the water contact angle is shown in Figure 5. When the contact angle changes from $95^{\circ}$ to $150^{\circ}$, the associated water-entry pressure increases from $2.8 \mathrm{MPa}$ to 28 MPa. The increase of the water-entry pressure with the water contact angle $\theta$ can be fitted to 316 capillary tube.

317 Figure 6 shows the dependence of the water saturation and the capillary pressure for contact 318 angles of $130^{\circ}$ and $150^{\circ}$ for water, respectively. The accordant curves suggested that for this model 319 there is little noticeable change to both node and bond filling orders at such high contact angles. 320 Therefore, the results simulated from one contact angle can be used to predict the results for other 321 contact angles. Note that the accordance of curves is not expected in general as the order of the 322 filling events for each node cannot be pre-determined and depends on the fluid configurations of all 323 connected bonds (see [36] and references therein). Figure 7 shows the relative permeability for the 324 liquid water versus the capillary pressure where the curve for the contact angle of $130^{\circ}$ was 325 calculated from the network model in comparison with the predicted from the capillary data 
simulated for the contact angle of $150^{\circ}$. Because of the impact of the Knudsen diffusion, the summation of the relative permeability for the air and liquid water is not unity.

Form the results above, it is clear that nanometre pores in the non-cracked MPL are capillary barriers to the water but paths to gaseous oxygen in which the Knudsen diffusion is significant [42]. Similar to the experimental work of Pant et al [24], we simulated the movement of oxygen, hydrogen and helium in the extracted pore network and calculated the apparent gas permeability for them. Table 2 shows the apparent gas permeability for the three gases as well as their Knudsen diffusion coefficients calculated at the same temperature of $299^{\circ} \mathrm{K}$. The mean-free-path between two consecutive collisions for oxygen, helium and hydrogen are $3.61 \times 10^{-10}(\mathrm{~m}), 2.18 \times 10^{-10}(\mathrm{~m})$ and $2.74 \times 10^{-10}(\mathrm{~m})$, respectively. Because the Knudsen number changes with the molecular properties of the gas, the associated apparent gas permeability increases with a decrease in molecular weight as expected. The oxygen permeability is nine times of the water permeability.

\section{Discussion and Remarks}

The calculated permeability for the liquid water in the MPL without cracks is in the order of $10^{-17} \mathrm{~m}^{2}$, smaller than the permeability reported in Pant et al [24], which was in the order of $10^{-13}$ $\mathrm{m}^{2}$, for their MPL which was also made from black carbon and PTFE. Their MPL permeability was measured indirectly by comparing the permeability of two GDLs with and without an MPL. The

343 permeability of GDLs reported in the literature is in the order of $10^{-11} \mathrm{~m}^{2}$, and the GDL

344 permeability measured by Pant et al. was also in this range. It is well-known that for a cylindrical 345 pore throat with a radius of $r$ its permeability is proportional to $r^{2}$. Pant et al. did not present the 346 pore sizes of their MPL, but from their estimated Knudsen numbers for their GDL and MPL, it can 347 be estimated that the average pore size in their MPL should be three orders of magnitude smaller 348 than that in the GDL; these were also consistent with the MPL we investigated in this paper and the 349 GDL we studied previously [43, 44]. The average pore size in our GDL and MPL were 13 microns and 30 nanometres, respectively, and the permeability of the GDL in both in-plane and through- 
351 plane directions is in the order of $10^{-11} \mathrm{~m}^{2}$. Given the effect of the Knudsen diffusion on the liquid 352 water being negligible, the permeability of the MPL for the liquid water should be in the order of $35310^{-17} \mathrm{~m}^{2}$ rather than $10^{-13} \mathrm{~m}^{2}$ as reported in Pant et al.. One reason for such a large discrepancy is 354 that the MPL permeability measured by Pant et al. could be for a gas, and the associated Knudsen numbers were approximately 2 . In this case, the gas permeability is not a constant but depends on the gas pressure and Knudsen number, and it is higher than the liquid water permeability. However, the difference in the Knudsen number for the water and gases alone still cannot explain that large difference in their permeability. The most likely reason is that there might be cracks in the MPL used by Pant et al. As mentioned previously crack development is a common phenomenon in MPLs. Martinez-Rodriguez et al. [13] showed that the aperture of the cracks was in the range of 4.4 to 32 361 microns estimated on the SEM images of MPLs. Furthermore, the density and aperture of the cracks appear to increase as the hydrophobicity increases [14]. In a hydrophobic media, big pores have less water-entry pressure and are much easier for the water to move through. The cracks hence provide preferential pathways for the liquid water to flow and substantially increase the permeability of MPLs. The impact of a set of cracks on the permeability of a low-permeability intact MPL can easily be illustrated analytically. For an MPL let the area-fraction of cracks be $\varepsilon$ and the areafraction of the remaining non-cracked be $(1-\varepsilon)$. When a fluid flows in the through-plane direction in a saturated matrix, there is little interference between the fluid in different cracks and the fluid in the MPL nanometre pores. Hence the averaged absolute permeability of a cracked MPL in the through-plane direction, $k$, consists of a permeability for cracks, $k_{\text {crack }}$, and a permeability for nano-

371 scale pores, $k_{M P L}$, that is, $k=\varepsilon k_{c r a c k}+(1-\varepsilon) k_{M P L}$. For the simplicity of this analysis, we assume that 372 the aperture of all cracks is $h$, and therefore the permeability of a single crack is $h^{2} / 12$. Take the 373 low bound of crack apertures reported in Martinez-Rodriguez, which was 4.5 microns, and the 374 permeability of a cracked MPL is $k=1.7 \times 10^{-12} \varepsilon+(1-\varepsilon) k_{M P L}\left(\mathrm{~m}^{2}\right)$. So even with $1 \%$ of the MPL 375 area being cracked, its contribution to the MPL permeability is in the order of $10^{-13} \mathrm{~m}^{2}$, much higher 
than the permeability of the nanometre pores in the MPL, which is in order of $10^{-17} \mathrm{~m}^{2}$. Taking the cracks into account could bring our results in consistency with the measurement of Pant et al. [24]. This reveals that the cracks developed in the strongly hydrophobic MPL would be the dominant pathways for the liquid water from the CL to the GDL, and the water fingering in the GDL observed in [45] is likely to be related to the cracks.

Figure 8 illustrates the possible water movement induced by the hydrophobic MPL. The MPL functions as a barrier to the water and the water condensed in the CL underneath the non-cracked MPL builds up a pressure first. Instead of pushing the water into the MPL, this increased pressure forces water moving backwards into the membrane as the $\mathrm{CL}$ is less hydrophobic; in the meantime, it also pushes the condensed water flowing laterally along the MPL-CL interface into the adjacent cracks, through which it migrates into the GDL. The nanometre pores in the MPL play only a minor role in the liquid water flow, but are the main pathways for oxygen to diffuse from the GDL to the CL. This is consistent with recent experimental observations that liquid water indeed moves through the cracks in the MPL rather than the nanometre pores [10,46]. This implies that although the cracks might accelerate MPL degradation, they appear to be beneficial to the water management.

Given the importance of pore structures and surface properties of an MPL on controlling gas and water flow behaviours, and the performance and durability of a PEM fuel cell, such relationships need to be better understood quantitatively than have ever been in order to improve the design of MPLs for better water management. As pointed out in a previous section, the sizedependent pore distribution in an MPL excludes scale and resolution limited techniques for investigating pore scale fluid transport directly. It is envisaged that multi-scale numerical modelling has a great potential to address 'what-if' questions concerning the water management in a costefficient manner. We, therefore, make the following remarks on developing multi-scale numerical models.

\section{Remark 1: modelling multi-modal flow behaviours}


Hydrophobic MPL pore systems may lead to bi-/tri-modal fluid flow behaviours balanced by viscous, capillary and gravity forces at different locations in time. Water mainly flows preferably through wide cracks. This may be driven mainly by the viscous force when the water pressure is above the capillary entry pressure and the pressure gradient is not negligible. The gas flows preferably in nanometre pores as their strong hydrophobicity and small diameters make it difficult for the water to overcome the capillary entry pressure if the water can be removed through the cracks. The water and gas flow behaviours could be more complex in micrometre pores as the water and gas may flow through them simultaneously, and the fractions of both phases may be equally significant at places in time. Micrometre pores, if connected to cracks, may function as a tributary; during water pressure build-up the water may be driven into those pores, pushing the gas back and

411 aside, while during water pressure draw-down, the gas may be imbibed into those water-filled pores, 412 along gas boundary layer, pushing back invading water or even 'swelling' at narrower throats to 413 snip off and trap the water phase.

414 Numerical modelling work on MPLs reported in the literature, however, ignores the complex 415 nature of multi-phase fluid dynamics, instead, deals with one aspect of the dynamics at a time, and 416 therefore neglects important multi-modal flow interactions. Because of aforementioned difficulties 417 in capturing diverse pore structures of an MPL sample, this problem is unlikely to be addressed by 418 any technique in use alone. Nevertheless, multi-scale imaging may offer a way to reconstruct a 419 multi-scale model of an MPL sample on which coupled crack flow, and micrometre and nanometre 420 pores network flow would be appropriate and may be modelled using similar techniques [47-49]. 421 Note that quasi-static pore-network models may not be sufficient to capture fluid dynamics when 422 the viscous force is not negligible. In this case dynamic pore network models would be required $423($ see $[50,51])$. 
Multi-scale imaging can be taken at selected sampling points at different resolutions to aid the reconstruction of an MPL. For example, one can use SEM to capture pore features on the surface of an MPL including cracks and large micro-pores and FIB/SEM, nano-XCT and micro-XCT to capture nanometre and micrometre pores. By registering multi-scale images together, aided by stochastic modelling to fill missing data, one could in principle generate a range of high-resolution models that capture a full range of pore types, sizes and distributions for an MPL [52]. When crack patterns can be treated as global features independently from local micro-/nano-scale pore structures and crack surface structures, we can extract pore networks from local volumes that do not contain cracks and populated over the non-cracked regions in a crack-pattern model.

For cracks that occur during drying and sintering processes of the MPL fabrication, the formations of crack patterns may depend on many factors as mentioned in a previous section. However, those patterns, in terms of crack intersecting angles and the area of partitioning, may closely resemble those of soils which undergo constrained desiccation. Therefore, it would be possible to model crack patterns stochastically as for soils [53]. An integrated pore network and crack model will enable investigations into the effects of crack patterns and composition of different 440 pores on gas and water transports.

\section{5. Conclusions}

This paper presented a method to calculate fluid flow properties of an intact MPL. A threedimensional microstructure of the MPL was acquired using the FIB/SEM tomography and the pore network was then extracted from a volume of $149^{3}$ voxels from a segmented image after being resampled at a resolution of $8 \mathrm{~nm}$ in voxel dimension. Water permeability values, which were

446 calculated from the extracted pore network using our pore network flow model and from the corresponding volume of the binary image using our lattice Boltzmann model, respectively, are 
449 enough to represent the MPL. We then calculated other flow properties on the extracted pore network.

The calculated absolute permeability for the liquid water is several orders of magnitude smaller than that reported in the literature. Further analysis indicated that such a discrepancy is likely due to the cracks developed in the MPL, as observed by many researchers. Such cracks did not appear in the volume of our MPL sample. Primary calculation shows that with only $1 \%$ of an MPL being cracked, its permeability could increase by two to five orders of magnitude, depending on the crack apertures. Given that the water entry pressure into the nanometre pores in the MPL is in the order of $10 \mathrm{MPa}$, it is very likely that the crack networks developed in an MPL are the dominant pathways for the liquid water to move from the CL to the GDL, and the nanometre pores in the MPL the 459 pathways for oxygen diffusion from the GDL to the CL.

There has been an exponential growth in research on PEM fuel cells over the past decade. Most 461 physical and electrochemical processes occurring in the cells are difficult to measure, and numerical modelling is therefore expected to play an important role in fuel cell design [54]. Success of these numerical models, however, relies on a detailed characterisation of the multi-scale MPL pore

464 system and an accurate description of each and all of important fluid flow processes. Since most components in the PEM fuel cells are opaque and only a few microns in size, experimentally

466 identifying mechanisms that control each transport process is difficult. The development in 467 tomography and pore-scale modelling has potential to bridge this gap as they have become 468 increasingly accessible and more accurate. This work is an attempt to use these technologies to 469 improve our understanding how an MPL may improve the water management in a PEM fuel cell, 470 and to suggest ways of modelling the multi-modal fluid flow effects in an MPL using an integrated 471 pore network and crack-pattern model.

472 Appendix A: Key facts on the construction of pore network from a 3D binary image

473 The pore network extraction procedure: 
1. Compute a Euclidean distance map for every pore voxel to its nearest solid voxel where this distance is the radius of the maximal inscribed ball centred at that pore voxel;

2. Transform the pore space into the medial axis (skeleton) by an improved thinning algorithm to

The reader is referred to [34] for detailed information on associated algorithms and methods.

\section{Appendix B: nomenclature for Equation 1}

\section{Description}




\begin{tabular}{|c|l|}
\hline$C_{g}$ & Correction factor for a circle, square or equilateral triangle and 0.5, 0.5623 and 0.6. \\
\hline$R_{h}$ & Hydraulic radius - the perimeter over the area of a cross-section \\
\hline$\rho_{2}$ & Density of gas at the outlet of an element \\
\hline$\mu_{2}$ & Viscosity of non-ideal gas at the outlet of an element \\
\hline$\mu_{0}$ & Reference viscosity of gas typically taken at 1 atmosphere \\
\hline$\mu_{2}^{i g}$ & Viscosity of ideal gas at the outlet of an element \\
\hline$N I G_{c}$ & The non-ideal gas coefficient for the continuum term \\
\hline$N I G_{k}$ & The non-ideal gas coefficient for the Knudsen term \\
\hline$\alpha$ & Tangential Momentum Accommodation Coefficient (TMAC) at pore wall \\
\hline$k_{n, 2 h}$ & Knudsen number \\
\hline$A$ & Area of the cross-section \\
\hline
\end{tabular}

\section{Acknowledgements}

The authors are grateful to two anonymous reviewers for their useful comments that helped to greatly improve the quality of our manuscript.

\section{References}

[1] X.L. Wang, H.M. Zhang, J.L. Zhang, H.F. Xu, Z.Q. Tian, J. Chen, H.X. Zhong, Y.M. Liang, (2006) 4909-4915.

[2] Z.G. Qi, A. Kaufman, Improvement of water management by a microporous sublayer for PEM fuel cells, J. Power Sources, 109 (2002) 38-46.

[3] R.P. Ramasamy, E.C. Kumbur, M.M. Mench, W. Liu, D. Moore, M. Murthy, Investigation of macro- and micro-porous layer interaction in polymer electrolyte fuel cells, Int. J. Hydrog. Energy, 33 (2008) 3351-3367.

[4] J.T. Gostick, M.A. Ioannidis, M.W. Fowler, M.D. Pritzker, On the role of the microporous layer in PEMFC operation, Electrochem. Commun., 11 (2009) 576-579.

[5] F.B. Weng, C.Y. Hsu, M.C. Su, Experimental study of micro-porous layers for PEMFC with gradient hydrophobicity under various humidity conditions, Int. J. Hydrog. Energy, 36 (2011) 13708-13714.

[6] R. Schweiss, M. Steeb, P.M. Wilde, Mitigation of Water Management in PEM Fuel Cell Cathodes by Hydrophilic Wicking Microporous Layers, Fuel Cells, 10 (2010) 1176-1180.

[7] P. Sung Bum, K. Sungjin, P. Yong-il, O. Myung-Hoon, Fabrication of GDL microporous layer using PVDF for PEMFCs, Journal of Physics: Conference Series, 165 (2009) 012046.

[8] N. Furuya, N. Mineo, Preparation of a Concentrated Carbon Black-PTFE Dispersion for Microporous Layer of PEMFC, Journal of New Materials for Electrochemical Systems, 10 (2007) 205. [9] S. Park, Y.-i. Park, Fabrication of gas diffusion layer (GDL) containing microporous layer using flourinated ethylene prophylene (FEP) for proton exchange membrane fuel cell (PEMFC), Int. J. Precis. Eng. Manuf., 13 (2012) 1145-1151.

[10] P. Deevanhxay, T. Sasabe, S. Tsushima, S. Hirai, Observation of dynamic liquid water transport in the microporous layer and gas diffusion layer of an operating PEM fuel cell by highresolution soft X-ray radiography, J. Power Sources, 230 (2013) 38-43. 
[11] C.-J. Tseng, S.-K. Lo, Effects of microstructure characteristics of gas diffusion layer and microporous layer on the performance of PEMFC, Energy Conversion and Management, 51 (2010) 677-684. transport within the cracks of micro porous layer in PEMFC, Electrochem. Commun., 13 (2011) 638-641.

[13] M.J. Martinez-Rodriguez, T. Cui, S. Shimpalee, S. Seraphin, B. Duong, J.W. Van Zee, Effect of microporous layer on MacMullin number of carbon paper gas diffusion layer, J. Power Sources, 207 (2012) 91-100.

[14] M. Ahn, Y.H. Cho, J. Kim, N. Jung, Y.E. Sung, Influence of hydrophilicity in micro-porous layer for polymer electrolyte membrane fuel cells, Electrochim. Acta, 56 (2011) 2450-2457. [15] W. Zhang, C.-w. Wu, Effect of Clamping Load on the Performance of Proton Exchange Membrane Fuel Cell Stack and Its Optimization Design: A Review of Modeling and Experimental Research, Journal of Fuel Cell Science and Technology, 11 (2013) 020801-020801. [16] Y. Matsui, T. Suzuki, P. Deevanhxay, S. Tsushima, S. Hirai, Crack Generation in Catalyst Layer and Micro Porous Layer by Wet-Dry Cycles and its Impact on PEMFC Performance, in: ASME 2013 11th International Conference on Fuel Cell Science, Engineering and Technology collocated with the ASME 2013 Heat Transfer Summer Conference and the ASME 2013 7th International Conference on Energy Sustainability, American Society of Mechanical Engineers, 2013, pp. V001T001A005-V001T001A005.

[17] H. Ostadi, P. Rama, Y. Liu, R. Chen, X.X. Zhang, K. Jiang, 3D reconstruction of a gas diffusion layer and a microporous layer, Journal of Membrane Science, 351 (2010) 69-74.

[18] E.A. Wargo, V.P. Schulz, A. Cecen, S.R. Kalidindi, E.C. Kumbur, Resolving macro- and micro-porous layer interaction in polymer electrolyte fuel cells using focused ion beam and X-ray computed tomography, Electrochim. Acta, 87 (2013) 201-212.

[19] H. Ostadi, P. Rama, Y. Liu, R. Chen, X. Zhang, K. Jiang, Nanotomography based study of gas diffusion layers, Microelectron. Eng., 87 (2010) 1640-1642.

[20] E.A. Wargo, T. Kotaka, Y. Tabuchi, E.C. Kumbur, Comparison of focused ion beam versus nano-scale X-ray computed tomography for resolving 3-D microstructures of porous fuel cell materials, J. Power Sources, 241 (2013) 608-618.

[21] J. Owejan, J. Owejan, T. Tighe, W. Gu, M. Mathias, Investigation of fundamental transport mechanism of product water from cathode catalyst layer in PEMFCs, in: ASME/JSME 2007 5th Joint Fluids Engineering Conference, American Society of Mechanical Engineers, 2007, pp. 13611367.

[22] H. Markötter, I. Manke, R. Kuhn, T. Arlt, N. Kardjilov, M.P. Hentschel, A. Kupsch, A. Lange, C. Hartnig, J. Scholta, J. Banhart, Neutron tomographic investigations of water distributions in polymer electrolyte membrane fuel cell stacks, J. Power Sources, 219 (2012) 120-125.

[23] P. Deevanhxay, T. Sasabe, S. Tsushima, S. Hirai, Observation of dynamic liquid water transport in the microporous layer and gas diffusion layer of an operating PEM fuel cell by highresolution soft X-ray radiography, Journal of Power Sources, 230 (2013) 38-43.

[24] L.M. Pant, S.K. Mitra, M. Secanell, Absolute permeability and Knudsen diffusivity measurements in PEMFC gas diffusion layers and micro porous layers, J. Power Sources, 206 (2012) 153-160.

[25] I.S. Hussaini, C.Y. Wang, Measurement of relative permeability of fuel cell diffusion media, J. Power Sources, 195 (2010) 3830-3840.

[26] U. Pasaogullari, C.Y. Wang, Two-phase transport and the role of micro-porous layer in polymer electrolyte fuel cells, Electrochim. Acta, 49 (2004) 4359-4369.

[27] M. Rebai, M. Prat, Scale effect and two-phase flow in a thin hydrophobic porous layer. Application to water transport in gas diffusion layers of proton exchange membrane fuel cells, J. Power Sources, 192 (2009) 534-543. 
[28] T. Inamuro, T. Ogata, S. Tajima, N. Konishi, A lattice Boltzmann method for incompressible two-phase flows with large density differences, J. Comput. Phys., 198 (2004) 628-644.

[29] H.W. Zheng, C. Shu, Y.T. Chew, A lattice Boltzmann model for multiphase flows with large density ratio, J. Comput. Phys., 218 (2006) 353-371. times, boundary conditions and the Knudsen layer, International Journal of Computational Fluid Dynamics, 22 (2008) 465-473.

[31] R. Wu, Q. Liao, X. Zhu, H. Wang, Y.D. Ding, J. Li, D.D. Ye, A Two-dimensional Network Study on Oxygen Transport in Porous Gas Diffusion Layer, Fuel Cells, 11 (2011) 255-262. [32] R. Wu, X. Zhu, Q.A. Liao, H. Wang, Y.D. Ding, J. Li, D.D. Ye, A pore network study on water distribution in bi-layer gas diffusion media: Effects of inlet boundary condition and microporous layer properties, Int. J. Hydrog. Energy, 35 (2010) 9134-9143.

[33] M. El Hannach, M. Prat, J. Pauchet, Pore network model of the cathode catalyst layer of proton exchange membrane fuel cells: Analysis of water management and electrical performance, Int. J. Hydrog. Energy, 37 (2012) 18996-19006.

[34] Z. Jiang, K. Wu, G. Couples, M.I.J. Van Dijke, K.S. Sorbie, J. Ma, Efficient extraction of networks from three-dimensional porous media, Water Resour. Res., 43 (2007).

[35] A. Ryazanov, M.I.J. van Dijke, K.S. Sorbie, Two-Phase Pore-Network Modelling: Existence of Oil Layers During Water Invasion, Transp. Porous Media, 80 (2009) 79-99.

[36] P.H. Valvatne, M.J. Blunt, Predictive pore-scale modeling of two-phase flow in mixed wet media, Water Resour. Res., 40 (2004).

[37] J. Ma, J.P. Sanchez, K. Wu, G.D. Couples, Z. Jiang, A pore network model for simulating nonideal gas flow in micro- and nano-porous materials, Fuel, 116 (2014) 498-508.

[38] P.E. Oren, S. Bakke, O.J. Arntzen, Extending Predictive Capabilities to Network Models, (1998).

[39] X.X. Zhang, L.K. Deeks, A.G. Bengough, J.W. Crawford, L.M. Young, Determination of soil hydraulic conductivity with the lattice Boltzmann method and soil thin-section technique, J. Hydrol., 306 (2005) 59-70.

[40] Y. Gao, X.X. Zhang, P. Rama, R. Chen, H. Ostadi, K. Jiang, An Improved MRT Lattice Boltzmann Model for Calculating Anisotropic Permeability of Compressed and Uncompressed Carbon Cloth Gas Diffusion Layers Based on X-Ray Computed Micro-Tomography, Journal of Fuel Cell Science and Technology, 9 (2012).

[41] J.H. Chun, K.T. Park, D.H. Jo, J.Y. Lee, S.G. Kim, S.H. Park, E.S. Lee, J.Y. Jyoung, S.H. Kim, Development of a novel hydrophobic/hydrophilic double micro porous layer for use in a cathode gas diffusion layer in PEMFC, Int. J. Hydrog. Energy, 36 (2011) 8422-8428.

[42] C. Chan, N. Zamel, X.G. Li, J. Shen, Experimental measurement of effective diffusion coefficient of gas diffusion layer/microporous layer in PEM fuel cells, Electrochim. Acta, 65 (2012) 13-21.

[43] Y. Gao, X.X. Zhang, P. Rama, Y. Liu, R. Chen, H. Ostadi, K. Jiang, Modeling Fluid Flow in the Gas Diffusion Layers in PEMFC Using the Multiple Relaxation-time Lattice Boltzmann Method, Fuel Cells, 12 (2012) 365-381.

[44] P. Rama, Y. Liu, R. Chen, H. Ostadi, K. Jiang, Y. Gao, X. Zhang, D. Brivio, P. Grassini, A Numerical Study of Structural Change and Anisotropic Permeability in Compressed Carbon Cloth Polymer Electrolyte Fuel Cell Gas Diffusion Layers, Fuel Cells, 11 (2011) 274-285.

[45] J.H. Kang, K.N. Kim, J.H. Nam, C.J. Kim, Visualization experiments and pore network simulations for invasion-percolation transport of a non-wetting fluid through multiple porous layers, Int. J. Hydrog. Energy, 37 (2012) 1642-1652.

[46] J.H. Chun, D.H. Jo, S.G. Kim, S.H. Park, C.H. Lee, S.H. Kim, Improvement of the mechanical durability of micro porous layer in a proton exchange membrane fuel cell by elimination of surface cracks, Renew. Energy, 48 (2012) 35-41. 
[47] R. Therrien, E.A. Sudicky, Three-dimensional analysis of variably-saturated flow and solute transport in discretely-fractured porous media, J. Contam. Hydrol., 23 (1996) 1-44. [48] M.T. Balhoff, K.E. Thompson, M. Hjorts $\emptyset$, Coupling pore-scale networks to continuum-scale models of porous media, Computers \& Geosciences, 33 (2007) 393-410.

[49] J. Chu, B. Engquist, M. Prodanović, R. Tsai, A Multiscale Method Coupling Network and Continuum Models in Porous Media I: Steady-State Single Phase Flow, Multiscale Modeling \& Simulation, 10 (2012) 515-549.

[50] V. Joekar-Niasar, S.M. Hassanizadeh, Analysis of Fundamentals of Two-Phase Flow in Porous Media Using Dynamic Pore-Network Models: A Review, Critical Reviews in Environmental Science and Technology, 42 (2012) 1895-1976. [51] I. Bondino, S.R. McDougall, G. Hamon, Pore-Scale Modelling of the Effect of Viscous Pressure Gradients During Heavy Oil Depletion Experiments, (2011). [52] S. Latham, T. Varslot, A. Sheppard, Image registration: Enhancing and calibrating X-ray micro-CT imaging, in: Proceedings of the International Symposium of the Society of Core Analysts. SCA2008-35. Abu Dhabi, United Arab Emirates, 2008.

[53] G.W. Horgan, I.M. Young, An empirical stochastic model for the geometry of twodimensional crack growth in soil (with Discussion), Geoderma, 96 (2000) 263-276.

[54] A.A. Shah, K.H. Luo, T.R. Ralph, F.C. Walsh, Recent trends and developments in polymer electrolyte membrane fuel cell modelling, Electrochim. Acta, 56 (2011) 3731-3757. 
653 Table 1 Comparison between the pore-network permeability and the image permeability calculated 654 using a lattice Boltzmann model.

\begin{tabular}{|l|c|c|}
\hline & $\begin{array}{c}\text { Image permeability } \\
\left(10^{-17} \mathrm{~m}^{2}\right)\end{array}$ & $\begin{array}{c}\text { Network permeability } \\
\left(10^{-17} \mathrm{~m}^{2}\right)\end{array}$ \\
\hline Through-plane direction & 5.08 & 1.82 \\
\hline In-plane direction (1) & 1.35 & 1.87 \\
\hline In-plane direction (2) & 1.60 & \\
\hline
\end{tabular}

657 Table 2 Apparent gas permeability for the three gases $\left(k_{g a s}\right)$ in comparison with the permeability for 658 liquid water $\left(k_{s}\right) . D_{p}$ is the Knudsen diffusion coefficient.

\begin{tabular}{|c|c|c|l|}
\hline Gas & $k_{\text {gas }}\left(\mathrm{m}^{2}\right)$ & $k_{\text {gas }} / k_{s}$ & $D_{p}\left(\mathrm{~m}^{2} / \mathrm{s}\right)$ \\
\hline Oxygen & $1.99 \times 10^{-16}$ & 9.19884 & $1.46 \times 10^{-11}$ \\
\hline Helium & $5.05 \times 10^{-16}$ & 23.3573 & $3.83 \times 10^{-11}$ \\
\hline Hydrogen & $3.28 \times 10^{-16}$ & 15.1809 & $5.53 \times 10^{-11}$ \\
\hline
\end{tabular}




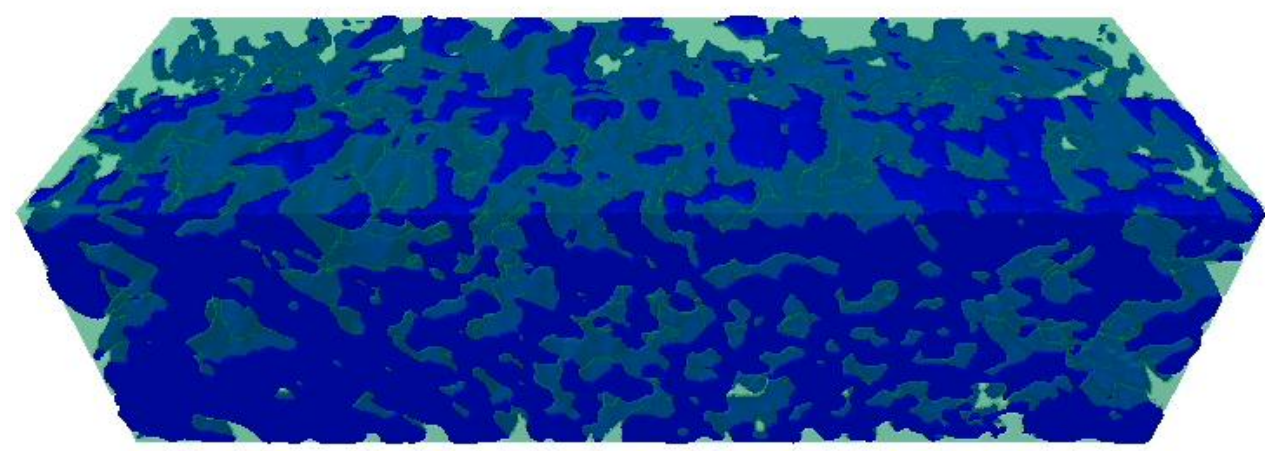

665 Figure 1. The reconstructed binary $3 \mathrm{D}$ volume corresponding to a volume of $1.5 \mu \mathrm{m} \times 1.5 \mu \mathrm{m} \times 5$ $666 \mu \mathrm{m}$ acquired from an MPL sample using FIB/SEM tomography (the blue is for solid and the gray 667 for pores).

668

669

670 


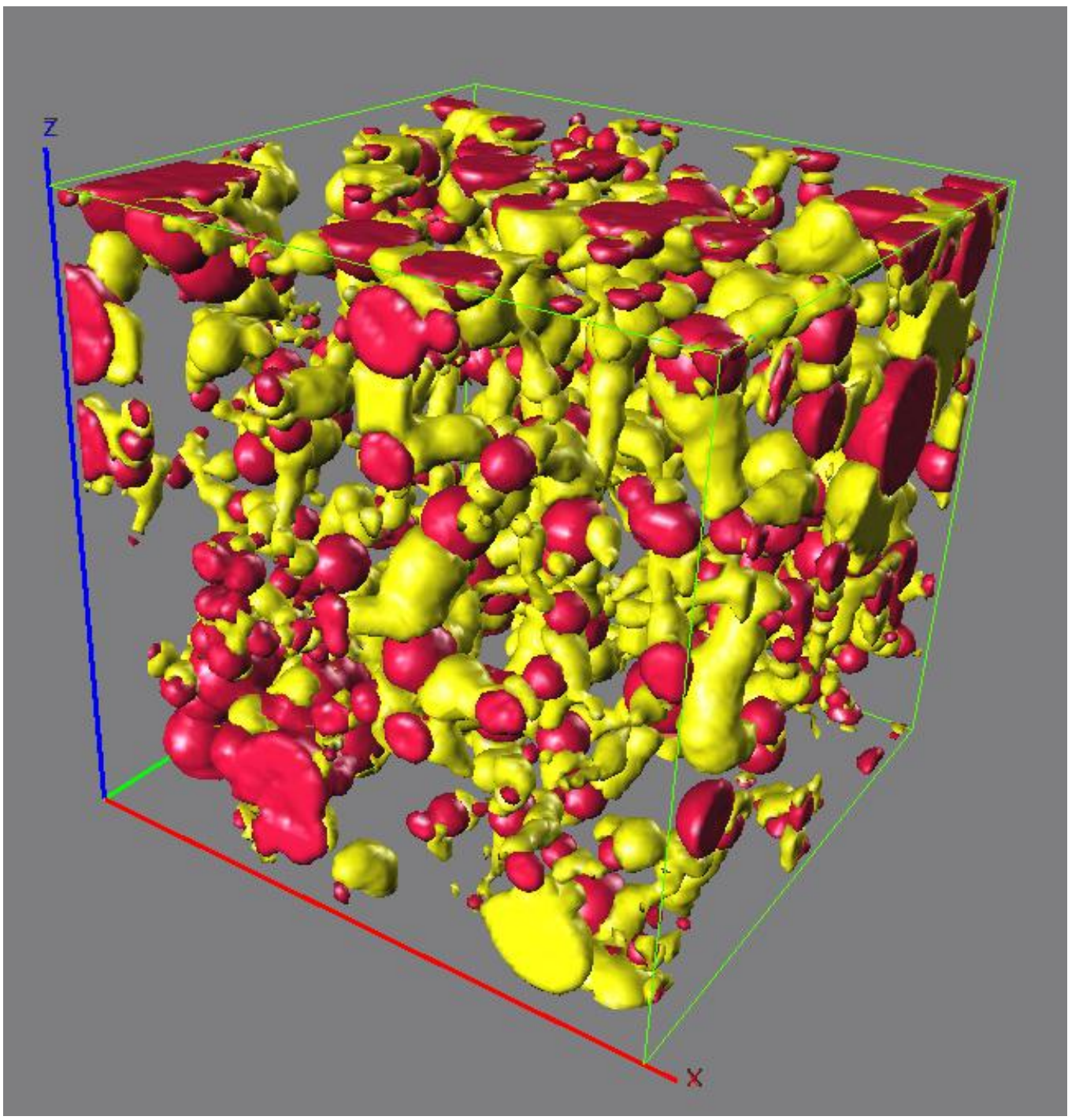

675

Figure 2. The partitioned pore space for the $149^{3}$ volume used in this work. Note that the voxel resolution is $8 \mathrm{~nm}$, and the node and bond volumes are in pink and yellow, respectively. 


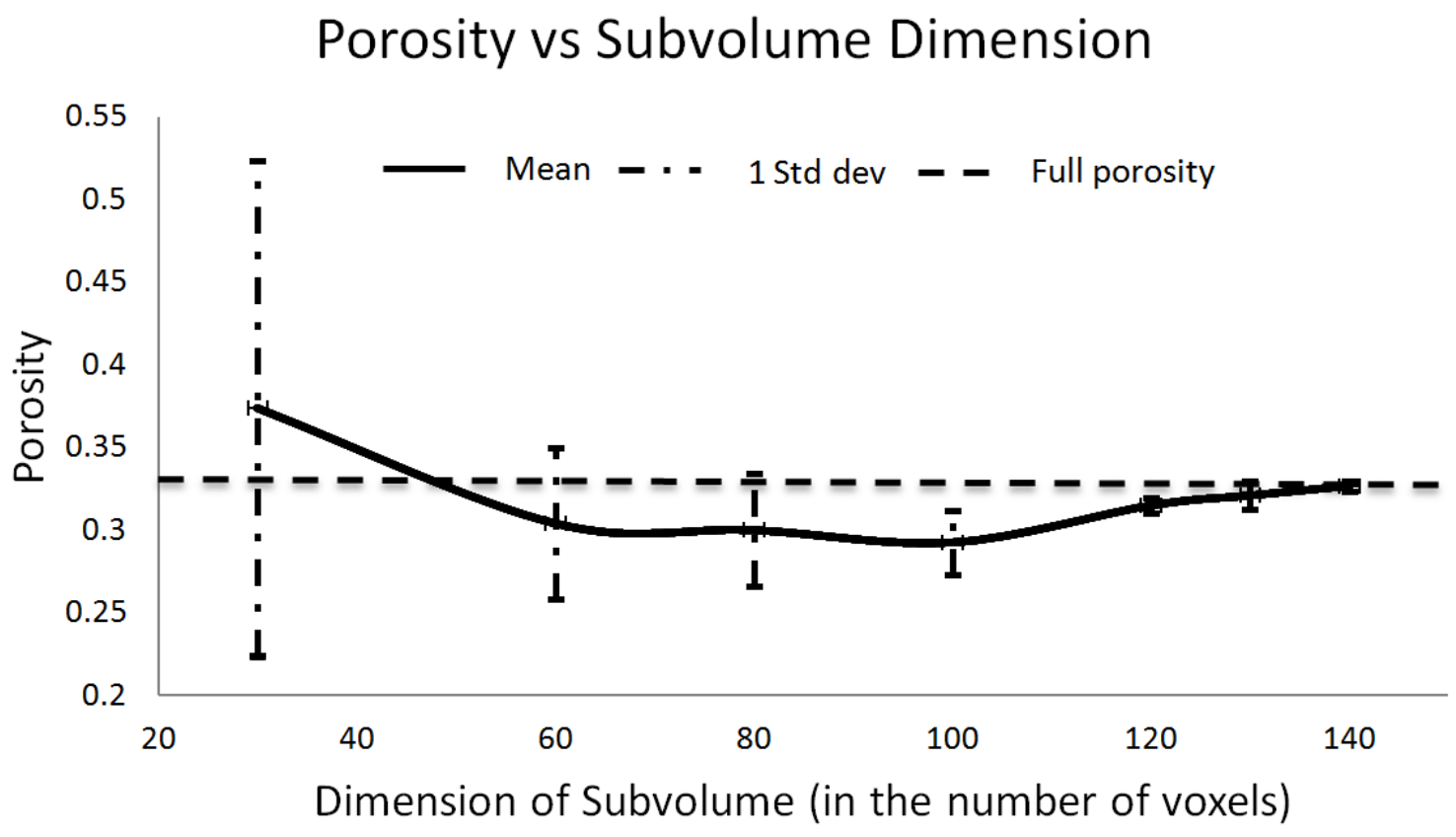

678 Figure 3. The mean porosity versus the dimension of sub-volume (the solid line) with errors of one 680 standard deviation drawn at both sides (dotted dash lines). The porosity of the selected volume is 681 about $34 \%$ and plotted as the dotted line for reference. 


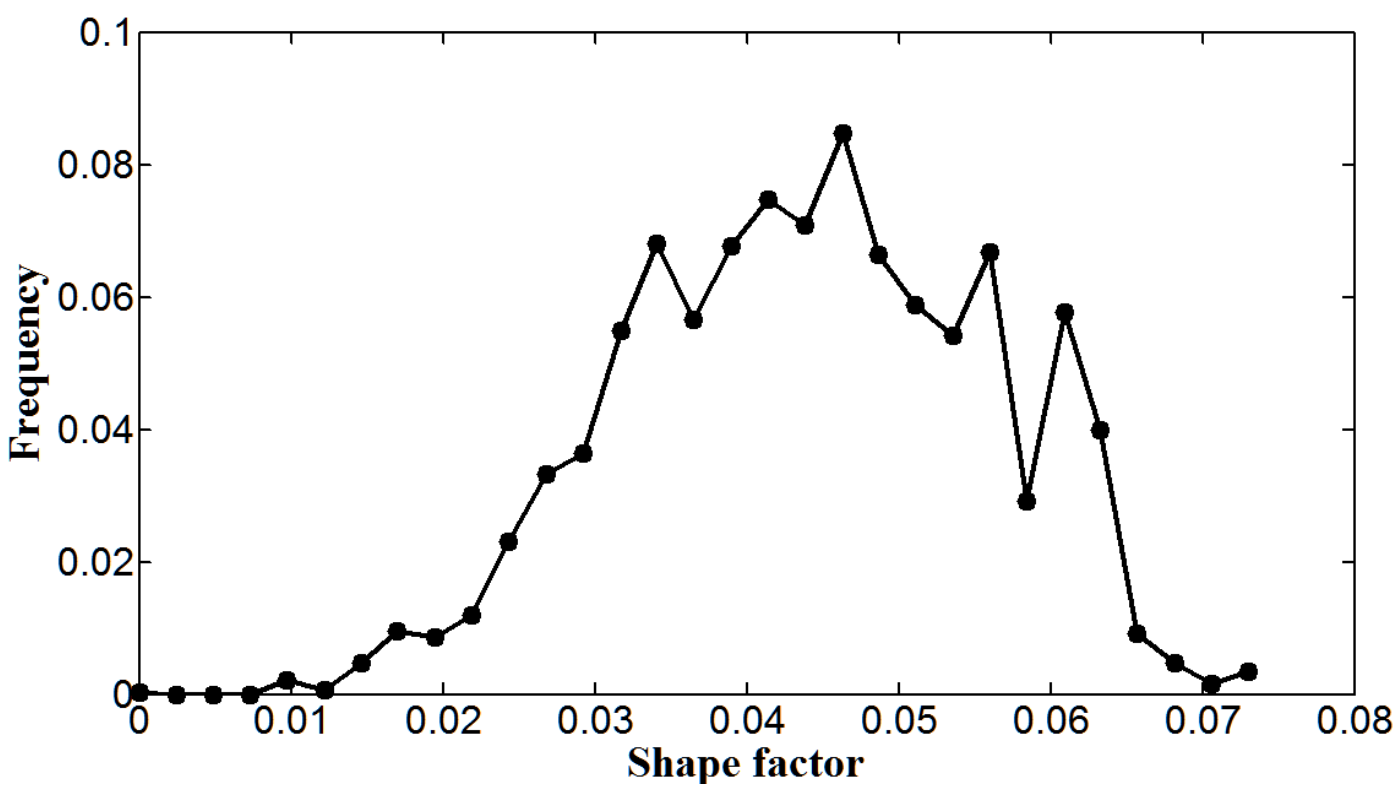

685

Figure 4. Frequency distribution of the shape factors calculated from the pore network. 


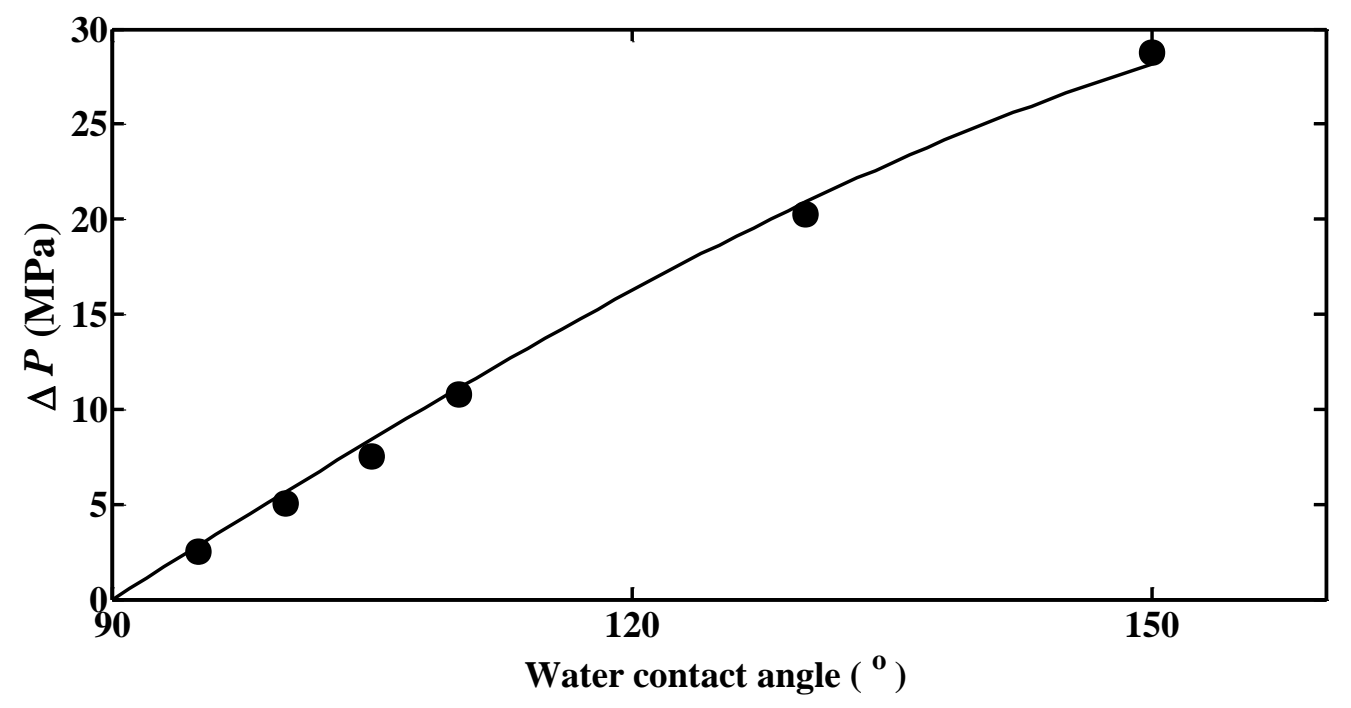

690

691 Figure 5. Increase of water-entry pressure with water contact angle. Symbols are simaultions and 692 the solid line is the fitting to $\Delta P=A \cos \theta$. 


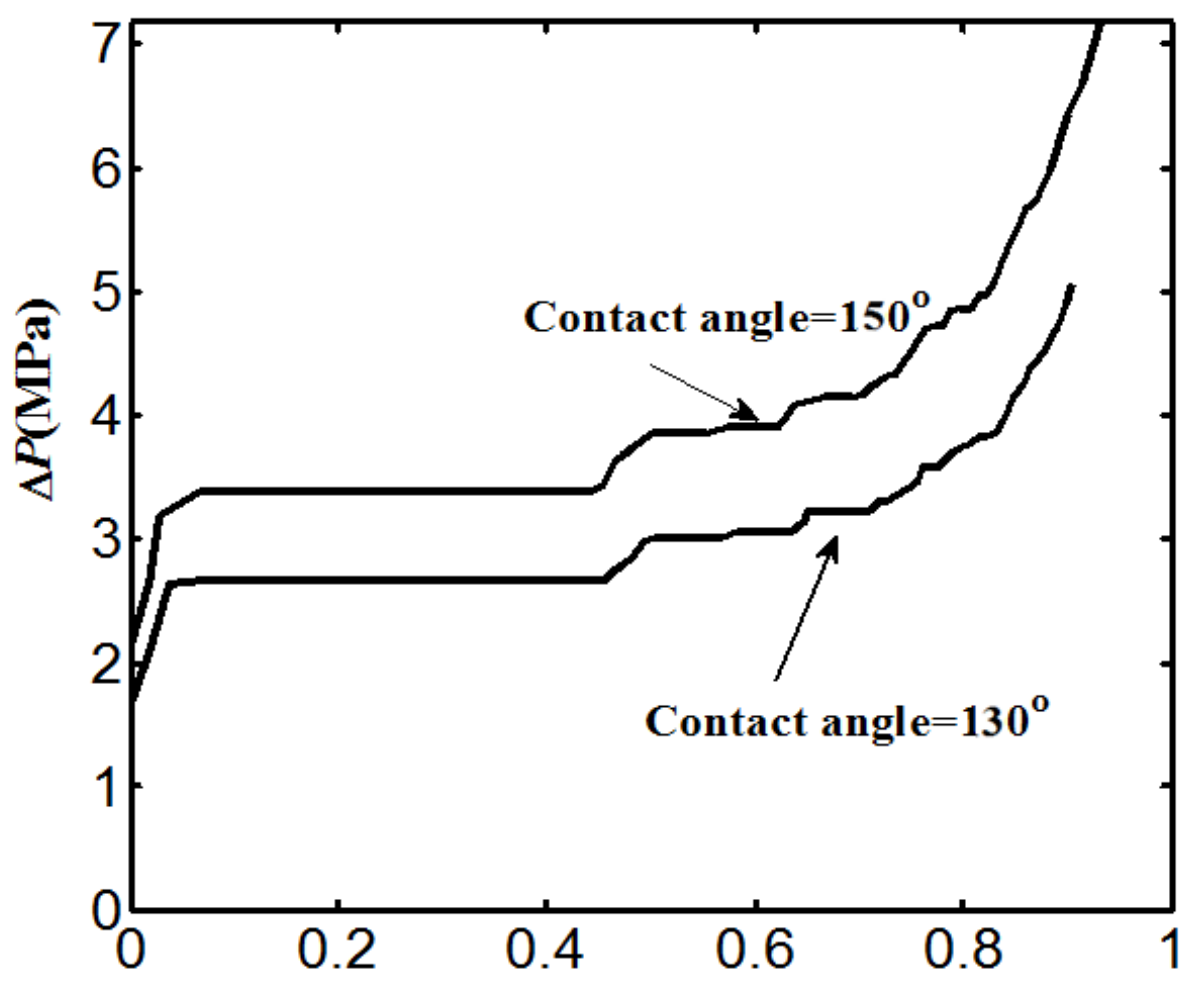

698 Figure 6. Impact of the water contact angle on the water saturation and the capillary pressure 699 relationship. 


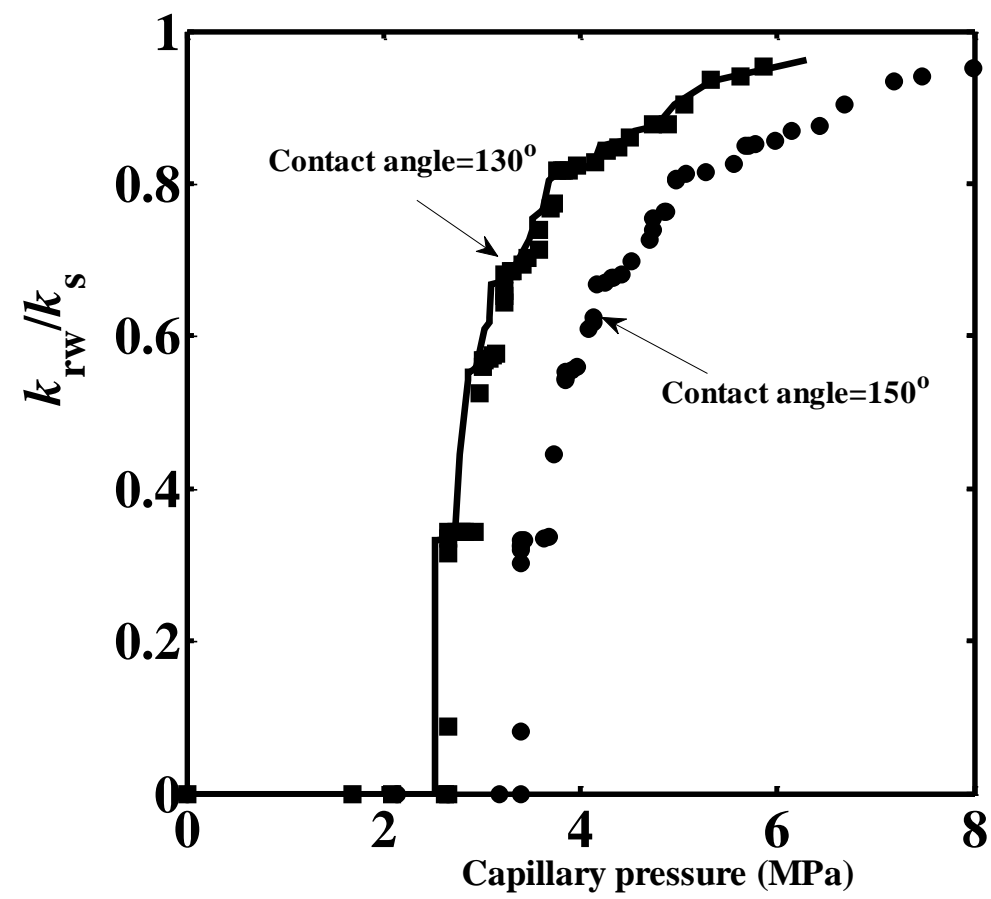

703 Figure 7. Change of relative permeability for liquid water with capillary pressure. Symbols are 704 simulations and the solid line is prediction using the results simulated with another contact angle.

Figure 8. Schematic illustration of liquid water movement in a cracked MPL. 